\title{
The critical role of the transition-state cusp diameter in understanding adiabatic and non-adiabatic electron transfer
}

\author{
Jeffrey R. Reimers ${ }^{\mathrm{ab}^{*}}$ and Noel S. Hush ${ }^{\mathrm{cd}^{*}}$ \\ a:Department of Physics and International Centre for Quantum and Molecular Structures, \\ Shanghai University, Shanghai 200444 China \\ b: School of Mathematical and Physical Sciences, University of Technology Sydney, NSW \\ 2007 Australia \\ c: School of Molecular Biosciences, The University of Sydney, NSW, 2006 Australia \\ d: School of Chemistry, The University of Sydney, Sydney, NSW 2006 Australia \\ * email: jeffrey.reimers@uts.edu.au, reimers@shu.edu.cn, noel.hush@sydney.edu.au
}

Keywords: electron transfer, transition state theory, cusp catastrophe, Born-Oppenheimer breakdown, non-adiabatic coupling, diabatic surfaces, Born-Huang surfaces, diagonal coupling

\begin{abstract}
The equation of Levich and Dogonadze describing the rate of electron-transfer processes in the weak-coupling "non-adiabatic" limit is understood in terms of the properties of general adiabatic electron-transfer theory. The cusp diameter describing the continuous changeover of Born-Oppenheimer adiabatic surfaces from donor-like to acceptor-like character is shown to be the critical property controlling reaction rates and intervalence spectra. Their work is presented in the context of general Born-Oppenheimer breakdown phenomena and linked to the overarching cusp catastrophe.
\end{abstract}




\section{INTRODUCTION}

In 1959, Levich and Dogonadze [1-4] deduced the rate constant for an electron-transfer reaction in the weak-coupling limit to be

$$
k=\frac{2 \pi}{\hbar} \frac{J^{2}}{\sqrt{4 \pi \lambda k_{\beta} T}} \exp \frac{-\Delta G^{\ddagger}}{k_{\beta} T}
$$

where $J$ is the electronic coupling linking the donor and acceptor, $\Delta G^{\ddagger}$ is the activation energy, $T$ the temperature, and $k_{\beta}$ Boltzmann's constant. This expression has subsequently been applied to understand electron-transfer processes in a very wide range of biological and nanotechnological applications [5, 6]. Standard transition state theory fails to take into account the probability $\kappa$, called the "transmission factor", that the reaction products form on the ground-state potential-energy surface and so needs to be corrected to

$$
k=\kappa \frac{\omega}{2 \pi} \exp \frac{-\Delta G^{\ddagger}}{k_{\beta} T},
$$

where $\omega$ is the collision frequency. Levich and Dogonadze deduced the value of the transmission factor following from the work of Kubo and Toyozawa [7] on rates of nonradiative processes treated only using Fermi's Golden Rule [8]. Their equation surpassed earlier crude quantum-tunnelling approaches [9-12] for estimating $\kappa$, exposing its dependence on experimentally assessable quantities. It is similar to results obtained in the same year by Holstein for related problems in condensed-matter physics [13, 14], following the work of Lax and others [15]. Figure 1 shows the key quantities involved in these approaches in the limit of small coupling. A feature of the Levich-Dogonadze derivation of the prefactor is that it is based on a single-mode model as illustrated in the figure, allowing Landau-Zener theory to be applied in the weak-coupling limit to obtain this analytical solution. Typically nuclear motions not involved in the reaction coordinate do contribute to reactivity, leading to deviations from Eqn. (1) [16-18], but nevertheless this equation remains the basis for modern conceptual understanding.

The activation energy $\Delta G^{\ddagger}$ appearing in Eqns. (1) and (2) had previously been determined by Kubo and Toyozawa [7] and by Hush [19, 20] in the limit of $J \rightarrow 0$ as the energy at the crossing point of the diabatic surfaces that occurs for harmonic potentials at geometry

$$
Q_{\mathrm{X}}=\frac{\Delta G_{0}}{\lambda} Q_{m}
$$

and is

$$
\Delta G^{\ddagger}=\frac{\left(\Delta G_{0}+\lambda\right)^{2}}{4 \lambda},
$$


where $\Delta G_{0}$ is the free-energy difference between products and reactants, $\lambda$ is the reorganization energy, and $Q= \pm Q_{m}$ specify the equilibrium geometries of non-interacting donor and acceptor species. The reorganization energy is the energy released when an optically induced vertically-excited charge-transfer state relaxes to its adiabatic energy minimum, given by $\lambda=2 \hbar \omega Q_{m}^{2}$ for harmonic potentials. Later an extended description of the basic equations and the motivations behind them was presented by Levich [21], forming a good summary of the work done with Dononadze.

The electronic coupling $J$ must always be non-zero or there would be no electron transfer. In Eqn. (1), its effect is treated perturbatively to manifest the reaction rate, but another effect is to modify the activation energy. For symmetric reactions with $\Delta G_{0}=0$, the activation energy is [22]

$$
\Delta G^{\ddagger}=\frac{(\lambda-2|J|)^{2}}{4 \lambda} .
$$

In general, it can be roughly approximated by [22]

$$
\Delta G^{\ddagger} \approx \frac{\left(\Delta G_{0}+\lambda-2|J|\right)^{2}}{4 \lambda} .
$$

As indicated clearly later [4], Levich and Dogonadze used quantum methods to treat the electronic motions that embody delocalization of the charge between the donor and the acceptor that changes continually as the electron-transfer process proceeds along the reaction coordinate. Such general quantum approaches are termed adiabatic electron transfer theory. They produced analytical results in two regimes: when the coupling is strong enough so that transition-state theory holds, and the limit of very weak coupling in which transition-state theory fails. In the weak-coupling limit, reactants cross transition states on BornOppenheimer adiabatic ground-state surfaces but fail to remain on the ground state, crossing instead to an excited state, and as a result this limit is often called the non-adiabatic limit. Equation (2) (with $\kappa=1$ ) and Eqn. (1) describe the transition-state theory results and the weak coupling results, respectively [4]. Both equations appear simple in that the shared quantum charge density does not need to be explicitly manifested and hence Levich and Dogonadze do not pursue the role of charge delocalization in their works. However, a different approach to adiabatic electron transfer theory had been introduced earlier by Hush $[19,20]$ that instead takes the quantum delocalization of charge as the central qualitative variable depicting electron transfer processes. Its use provides a simple conceptual link between the two analytically solvable cases considered by Levich and Doganadze.

In this article, we consider the physical insight into the Levich-Dogonadze equation that can be gained using Hush's approach, utilizing modern interpretations of key concepts not available to 1950's researchers. While all methods for the calculation of rate constants using first-principles electronic-structure approaches are, by necessity, based on adiabatic 
electron-transfer theory, how electron-transfer processes relate to general chemical reactions, as well as to general non-adiabatic processes, is here of central concern.

\section{ADIABATIC ELECTRON-TRANSFER THEORY}

Adiabatic electron-transfer theory was introduced by Hush in 1956 [19, 20]. It flows directly from the general chemical kinetics models constructed using diabatic descriptions of reactants and products developed in the 1920's-30's [23-29]. Today diabatic models are in extremely widespread use for the qualitative and quantitative description of all types of processes throughout biochemistry, chemistry, and nanotechnology [30]. However, the success achieved when applied to electron-transfer problems was outstanding, allowing extremely diverse properties such as geometries, vibrational spectra, electronic spectra, electron-spin-resonance spectra, Stark spectra, rate constants, redox potentials, etc., all to be interpreted using the core model parameters $J, \lambda, \omega$, and $\Delta G_{0}$ [30-32]. How to extend such simple analysis to general chemical reactions has just been determined [30], demonstrating applications including: understanding aromaticity, determining why it is that the chemistry of the second row of the periodic table is so different to that of the first row [33], understanding general non-adiabatic reactions [22], and determining design principles for chemical quantum qubits for use in quantum information processing [34, 35].

The centerpiece of adiabatic electron-transfer theory is that the electronic coupling $J$ connects the uncoupled diabatic reactant and product potential-energy surfaces to make adiabatic surfaces with gradually changing properties. Initially, the electron-transfer problem is expressed in a donor (reactant) and acceptor (product) diabatic basis $\left\{\left|\psi_{\mathrm{D}}\right\rangle,\left|\psi_{\mathrm{A}}\right\rangle\right\}$ as

$$
\begin{aligned}
& \mathbf{H}=\left[\begin{array}{cc}
T+V_{D}(Q) & J \\
J & T+V_{A}(Q)
\end{array}\right] \text { where } \\
& V_{D}(Q)=\frac{\hbar \omega}{2}\left(Q+Q_{m}\right)^{2} \text { and } \\
& V_{A}(Q)=\frac{\hbar \omega}{2}\left(Q-Q_{m}\right)^{2}+\Delta G_{0} .
\end{aligned}
$$

Here, $V_{D}(Q)$ and $V_{A}(Q)$ are the donor and acceptor potential-energy surfaces, respectively, expressed as a function of a dimensionless normal coordinate $Q$ defined such that $Q=1$ corresponds to a displacement of one zero-point length $\sqrt{\hbar / \omega}$ in the direction of nuclear motion associated with the electron transfer. Also, $T$ is the nuclear kinetic-energy operator, $T=-(\hbar \omega / 2) \partial^{2} / \partial Q^{2}$, and $\pm Q_{m}$ specifies the equilibrium geometries of non-interacting donor and acceptor species. Some example adiabatic surfaces are shown in Figure 2, obtained using the Born-Oppenheimer approximation [36-38] to diagonalize $\mathbf{H}$ parametrically at each value of the nuclear coordinate $Q$. While early diabatic models focused on bond dissociation [23-28] and often used coupled Morse oscillators, the harmonic surfaces depicted here were introduced to simplify the analysis of oxidation-reduction processes [29] and soon became widely applied [1, 2, 7, 12-14, 19]. The diabatic description 
in Eqn. (7) is simple and intuitive, facilitating Levich and Doganodze's description [1-4] of electron transfer as an "ion to ion" process driven by the coupling $J$.

Diagonalization of the linearly-coupled harmonic-oscillator model depicted in Eqn. (7) produces two adiabatic surfaces $\varepsilon_{ \pm}(Q)$, as shown in Fig. 2., where\{Hush, $\left.1980 \# 319\right\}$

$$
\varepsilon_{ \pm}(Q)=\frac{\Delta G_{0}}{2}+\frac{\lambda}{4}+\frac{\hbar \omega}{2} Q^{2} \pm\left[\left(\frac{\Delta G_{0}}{2}-\hbar \omega Q_{m} Q\right)^{2}+J^{2}\right]^{1 / 2}
$$

The corresponding adiabatic wavefunctions $\left\{\left|\psi_{-}\right\rangle,\left|\psi_{+}\right\rangle\right\}$are expressed in terms of the eigenvectors of $\mathbf{H}$ as

$$
\begin{aligned}
& \left|\psi_{-}\right\rangle=a(Q)\left|\psi_{\mathrm{D}}\right\rangle+b(Q)\left|\psi_{\mathrm{A}}\right\rangle \\
& \left|\psi_{+}\right\rangle=-b(Q)\left|\psi_{\mathrm{D}}\right\rangle+a(Q)\left|\psi_{\mathrm{A}}\right\rangle
\end{aligned},
$$

where $\{$ Hush, $1980 \# 319\}$

$$
\begin{gathered}
a^{2}(Q)=\frac{1}{2}+\frac{\frac{\Delta G_{0}}{\lambda}-\frac{Q}{Q_{m}}}{2\left[\left(\frac{\Delta G_{0}}{\lambda}-\frac{Q}{Q_{m}}\right)^{2}+\left(\frac{2 J}{\lambda}\right)^{2}\right]^{1 / 2}} \text { and } \\
b^{2}(Q)=\frac{1}{2}-\frac{\frac{\Delta G_{0}}{\lambda}-\frac{Q}{Q_{m}}}{2\left[\left(\frac{\Delta G_{0}}{\lambda}-\frac{Q}{Q_{m}}\right)^{2}+\left(\frac{2 J}{\lambda}\right)^{2}\right]^{1 / 2}}=1-a^{2}(Q) .
\end{gathered}
$$

Electron-transfer reactions involve the transfer of charge from the donor to the acceptor, and $b^{2}(Q)$ tells the fraction of that charge transferred at each intermediate nuclear geometry as the reaction proceeds, whilst $a^{2}(Q)$ tells the fraction retained. The above steps are common to the electron-transfer approaches both of Levich and Dogonadze [1-4] and of Hush [19, 20], differing significantly from other approaches $[12,40]$ suggested at the time. From this point, Levich and Dogonadze applied approximations to deduce Eqns. (1) and (2) (with $\kappa=1$ ).

Hush, however, focused on the general nature of electron transfer. From Eqns. (9)(11), the fraction of charge transferred at the crossing of the diabatic states is $b^{2}\left(Q_{X}\right)$, which will be close to the fraction transferred at any transition state. As for all chemical reactions, the fraction of charge transferred at the transition state is critical for the understanding of intermolecular interactions with neighbouring solvent, biological, or internal atomic structures [41-45]. Modern recommendations for computational schemes to deduce the 
critical parameters $\Delta G_{0}, \Delta G^{\ddagger}$, and $|J|$ often involve calculations with implicit or explicit solvent performed at the geometries of both the adiabatic minima and the crossover point $Q_{X}$. Calculations performed at $Q_{X}$ explicitly manifest the charge transferred at the crossing point and hence provide optimal evaluation of $\Delta G^{\ddagger}[46]$.

Four classic example systems described by adiabatic electron-transfer theory are shown in the Fig. 2: two with weak coupling ( $2|J| / \lambda \ll 1$ ), typical of non-adiabatic electrontransfer processes in the "inverted" $\left(\left|\Delta G_{0}\right|>\lambda\right)[1,5,7,47]$ and "normal” $\left(\left|\Delta G_{0}\right|<\lambda\right)$ [19] regions; one with intermediate coupling in the normal region, typical of applications of transition-state theory; and one with strong coupling [48, 49], typical of delocalized "resonance" interactions such as that between the Kekulé structures of benzene [30]. A key feature is that the adiabatic surfaces change slowly in nature between reactants and products, avoiding the unphysical discontinuous changes in character central to non-adiabatic electrontransfer approaches. In 1932, London showed that this slow change in character was critical to system properties [24]. The process is illustrated in Fig. 3 and can be quantified through the introduction of the cusp diameter $Q_{C}$ [22]

$$
Q_{C}=\frac{2|J|}{\lambda} Q_{m}
$$

On the left-hand side of the figure, donor character dominates the ground adiabatic surface so that $a^{2}$, the fraction of donor character, is near 1 , falling continuously to near 0 on the righthand side. The main diagrams in Fig. 3 focus on the region near the transition state where the ground-state character changes quickly, whilst the zoomed-out insert presents a broader perspective. The derivatives $\mathrm{d}^{2} / \mathrm{d} Q$ and $\mathrm{d}^{2} a^{2} / \mathrm{d} Q^{2}$ are shown in the figure, with $Q_{C}$ defined as the distance between the minimum and maximum points in the second-derivative plot.

In the limit of weak coupling, the cusp diameter becomes very small, meaning that adiabatic transition-states (in the normal regime) become extremely sharp with associated extremely high imaginary barrier frequencies. However, when the coupling is large the cusp diameter can exceed the geometry difference between the "reactants" and "products", giving rise to a single ground-state structure, e.g., as is observed for benzene instead of one of the conceptualized Kekulé "reactant” and "product” structures. Adiabatic electron-transfer theory is therefore very broad in its applicability to chemical processes [30].

$Q_{C}$ is called the "cusp" diameter as, in the limit of $J \rightarrow 0$, a discontinuous cusp between reactants and products is produced, providing an example of a pitchfork-bifurcation cusp catastrophe [50-53]. The mathematics of such systems is in general complex, with chaos setting in as dynamics near a cusp is investigated. This chaos prevents simple approaches, using for instance perturbation theory, from describing the dynamics, with profound consequences for the understanding of the breakdown of the Born-Oppenheimer approximation [22]. As $J \rightarrow 0$, the two adiabatic potential-energy surfaces become 
degenerate and, when multiple nuclear coordinates are involved, a conical intersection seam is encountered. Equation (1) derived by Levich and Dogonadze therefore represents a significant result pertaining to cusp catastrophes. Hence the cusp diameter must play a significant role in providing physical insight for this equation. This applies to not just electron-transfer theory but also to general processes involving conical intersections as reactions typically proceed around them instead of through them and hence access finite coupling $J$ [54]. Its significance is most clearly seen through the nature of the Pseudo-JahnTeller effect model of chemical reactivity $[49,55,56]$.

\section{FAILURE OF THE BORN-OPPENHEIMER APPROXIMATION}

The Born-Oppenheimer approximation used in obtaining Eqn. (8) neglects the effects of the nuclear momentum and kinetic energy operators on the electronic wavefunctions, allowing description as functions of geometry only. Its errors scale naively as the quarter root of the ratio of the electronic and nuclear masses [36], something akin to $(\hbar \omega)^{1 / 2} /\left(4 J^{2}+\lambda^{2}\right)^{1 / 4}$. Equation (6) can be rewritten exactly [22, 37, 38] in the Born-Oppenheimer basis $\left\{\left|\psi_{-}\right\rangle,\left|\psi_{+}\right\rangle\right\}$as

$$
\mathbf{H}=\left[\begin{array}{cc}
T+\varepsilon_{-}(Q)+\Delta H^{D C}(Q) & \Delta P^{F D}(Q) \frac{\partial}{\partial Q}+\Delta H^{S D}(Q) \\
\Delta P^{F D}(Q) \frac{\partial}{\partial Q}+\Delta H^{S D}(Q) & T+\varepsilon_{+}(Q)+\Delta H^{D C}(Q)
\end{array}\right],
$$

identifying [22] three correction terms to the Born-Oppenheimer approximation

$$
\mathbf{H} \approx\left[\begin{array}{cc}
T+\varepsilon_{-}(Q) & 0 \\
0 & T+\varepsilon_{+}(Q)
\end{array}\right]
$$

as the momentum (or first derivative) correction

$$
\Delta P^{F D}(Q)=\frac{-\hbar^{2} \omega^{2} Q_{m} J}{2 J^{2}+\hbar \omega \lambda\left(Q-Q_{\mathrm{x}}\right)^{2}}=\frac{\hbar \omega}{2} \frac{Q_{C}}{Q_{C}^{2}+\left(Q-Q_{X}^{2}\right)},
$$

the diagonal correction

$$
\Delta H^{D C}(Q)=\frac{1}{2 \hbar \omega}\left(\Delta P^{F D}(Q)\right)^{2},
$$

shown graphically in Fig. 3, and the kinetic energy (or second derivative) correction

$$
\Delta H^{S D}(Q)=\frac{2}{\hbar \omega} \frac{Q-Q_{X}}{Q_{C}}\left(\Delta P^{F D}(Q)\right)^{2} .
$$

While the diabatic description of Eqn. (7) is a simple and intuitive one focusing on the intrinsic properties of the donor and acceptor chemical species and the coupling between 
them, the adiabatic representation Eqns. (13)-(16) present the same information in a complex form, with $\varepsilon_{ \pm}(Q)$ (Eqn. (8)) and $Q_{C}$ (Eqn. (12)) implicitly describing the effects of the coupling $J$. As $J \rightarrow 0$, Eqn. (7) produces simple physically intuitive solutions whilst $\varepsilon_{ \pm}(Q)$ develop a discontinuous cusp and, as also $Q_{C} \rightarrow 0$, the Born-Oppenheimer corrections $\Delta P^{F D}(Q), \Delta H^{S D}(Q)$, and $\Delta H^{D C}(Q)$ grow unboundedly large.

The inter-relationships between the different Born-Oppenheimer corrections evident in Eqns. (16)-(17) are examples of those known when more than one nuclear motion is included [57-60]. Noting that the Born-Oppenheimer approximation largely works well and that the diagonal and second-derivative corrections enter as the square of the first-derivative correction, it is tempting to assume that the first-derivative correction is more important and therefore that the others can be neglected. However, the chaotic nature of dynamics near a cusp means that this is not so [50-53]. Indeed, for a wide range of spectroscopic and kinetic properties we find, using full quantum solution to the associated nuclear dynamics, that there is no region of the parameter space of Eqn. (7) for which including only the first-derivative correction produces qualitatively realistic results for Born-Oppenheimer breakdown [22]. Nevertheless, this assumption is widely used in non-adiabatic dynamics calculations. Much effort is currently being spent in developing efficient methods for evaluating the diagonal correction [37, 61-90] and the second-derivative correction [58, 91, 92] for use in high-level quantum dynamics calculations of kinetics, thermodynamics, and spectroscopy.

In contrast, there are problems for which use of only the diagonal correction leads to realistic descriptions of Born-Oppenheimer breakdown [22]. Such problems often involve the region of the ground-state minimum-energy geometry and eventuate because the diagonal correction applies independent of the energy gap between the ground-state and excited-state Born-Oppenheimer surfaces, whereas the first-derivative and second-derivative corrections couple these states together and therefore fall off additionally as the energy gap increases. The diagonal correction term when added to the Born-Oppenheimer potential-energy surfaces produces what are known as Born-Huang adiabatic potential-energy surfaces [37, 38]

$$
\varepsilon_{ \pm}^{B H}(Q)=\varepsilon_{ \pm}(Q)+\Delta H^{D C}(Q) \text {. }
$$

Figure 2 shows these surfaces in addition to the usual Born-Oppenheimer ones. The effect of the diagonal correction (see Eqns. (15) and (16)) is to introduce a spike (see Fig. 3) at the location of the crossing of the original diabatic surfaces of height

$$
\Delta \Delta G^{\ddagger}=\left(\Delta H^{D C}(Q)\right)_{\max }=\frac{\hbar \omega}{8 Q_{C}^{2}}
$$

To a good approximation, a key effect of the diagonal correction is to increase the activation energy $\Delta G^{\ddagger}$ by this amount. When this term is large, the nuclei move too fast to allow the electrons to respond, trapping reactants in their initial diabatic (donor) state. 
Failure of traditional Born-Oppenheimer-based transition-state theory (Eqn. (2) with $\kappa=1$ ) to describe electron-transfer processes can therefore be interpreted, not so much as a failure of transition-state theory itself, but rather a failure caused by the use of the BornOppenheimer ground-state barrier height $\Delta G^{\ddagger}$ rather than the Born-Huang value $\Delta G^{\ddagger}+\Delta \Delta G^{\ddagger}$. The situation is not quite that simple, however, as the spike caused by the diagonal correction can be both very high $\left(\propto 1 / Q_{C}^{2}\right)$ and very narrow (width $\propto Q_{c}$ ) and hence nuclear tunnelling through the Born-Huang ground-state barrier must also be taken into account, adding a significant nuclear quantum effect.

When nuclear tunnelling through the ground-state potential-energy barrier is included into transition-state theory, a simple description of the significance of the diagonal correction can be made. Reactions in the inverted region are often classified as "non-adiabatic" as a radiationless transition is required to convert reactants to products, with the reactants and products described by different Born-Oppenheimer potential-energy surfaces. One is then concerned with the rate constants of surface-hopping reactions induced by $\Delta P^{F D}(Q)$ and $\Delta H^{S D}(Q)$. In the Born-Huang description of this scenario, mostly the point of inflection manifested in Fig. 2 for the inverted reaction disappears and is replaced by an adiabatic transition state linking reactants to products. The diagonal correction blocks electron-transfer by creating a high barrier, but the rate does not decrease exponentially with the barrier height as classical transition-state theory predicts because the nuclear tunnelling through the increasingly narrow barrier leads to only power-law scaling. In this way the critical features of the Levich-Dogonadze equation are produced, without the need to involve the second adiabatic surface. Many reactions deemed "non-adiabatic” with regard to Born-Oppenheimer surfaces are therefore "adiabatic" with regard to Born-Huang surfaces. Quantitative understanding, including the full derivation of the Levich-Dogonadze equation, requires the inclusion of the surface hopping between the Born-Huang description, however [22].

\section{UNDERSTANDING THE LEVICH-DONONATZE EQUATION}

All of these factors are incorporated into Eqn. (1) for the rate of electron-transfer reactions in the weak-coupling limit. It can simply be rewritten as

$$
k=\pi^{3 / 2}\left(\frac{\lambda}{k_{\beta} T}\right)^{1 / 2} Q_{C}^{2} \frac{\omega}{2 \pi} \exp \frac{-\Delta G^{\ddagger}}{k_{\beta} T},
$$

identifying the transmission factor $\kappa$ as

$$
\kappa=\pi^{3 / 2}\left(\frac{\lambda}{k_{\beta} T}\right)^{1 / 2} Q_{C}^{2}
$$

Hence the electron-transfer rate scales simply with the square of the cusp diameter- the sharper the cusp, the slower the rate. Using Eqn. (19), this could be written in terms of the key properties of the ground-state Born-Huang adiabatic potential-energy surface as 


$$
\kappa=\pi^{3 / 2}\left(\frac{\lambda}{k_{\beta} T}\right)^{1 / 2} \frac{\hbar \omega}{8 \Delta \Delta G^{\ddagger}}
$$

For chemical processes ranging from aromaticity through traditional reactions to fast electron-transfer processes pertinent to chemical and technological applications $(0.01<$ $2|J| / \lambda<10$ and $\left.0.003<\hbar \omega /\left(4 J^{2}+\lambda^{2}\right)^{1 / 2}<1\right), Q_{C}$ ranges over 6 orders of magnitude [22], increasing to 10 orders if weak electron-transfer processes in biology and photochemical charge recombination [93] are considered. Hence $\Delta \Delta G^{\ddagger}$ can vary by over 20 orders of magnitude, providing an important descriptor.

In practical applications of the Levich-Dogonadze equation, care must always be taken to assure that the parameters used generate $\kappa<1$, but this and various other key criteria are often neglected based on an empirical ansatz that many order-of-magnitude errors in its use typically cancel each other out $[94,95]$. As $\kappa$ increases towards 1 , the weak-coupling limit no longer applies but instead the Born-Oppenheimer approximation holds and traditional transition-state theory works. From Eqn. (22), using typical values of $\lambda$ of 0.25 to $1 \mathrm{eV}$ at room temperature, $\kappa<1$ requires

$$
Q_{C}<0.15 \text { to } 0.3 \text {. }
$$

However, we have previously observed empirically [22] that the Born-Oppenheimer approximation holds well whenever the diagonal correction maximum $\Delta \Delta G^{\ddagger}$ is less than one vibrational quantum in height, requiring

$$
Q_{C}>8^{-1 / 2} \approx 0.35
$$

Hence the Levich-Dogonadze equation provides another example of general aspects of the breakdown of the Born-Oppenheimer approximation.

Electron transfer in the weak coupling limit, as depicted by the Levich-Dogonadze equation, is usually termed a "non-adiabatic" process as it cannot be described using a single potential-energy surface within the Born-Oppenheimer adiabatic approximation. In practical calculations, both surfaces are usually included and coupled using the first-derivative nonadiabatic coupling operator. However, in numerical simulations [22], we find this approximation to be qualitatively useful in no region of the parameter space of the model. Instead, tunnelling dynamics on the single Born-Huang adiabatic ground-state potentialenergy surface is found to mostly give results in general agreement with the LevichDononatze equation [22]. While accurate solution of the electron-transfer problem requires full inclusion of all three corrections to the Born-Oppenheimer equation [22], Eqn. (22) implies an importance for the diagonal correction as it shows that the Levich-Dogonadze equation can be written explicitly in terms of the primary quantities of the Born-Huang surfaces. Taking a very simplistic view and treating the diagonal correction as adding a 
rectangular barrier of height $\Delta \Delta G^{\ddagger}$ and width $Q_{C}$ to the ground-state surface, the standard expression for nuclear barrier tunnelling at energy $E$ below the barrier becomes

$$
\kappa=\left[1+\frac{\Delta \Delta G^{\ddagger 2}}{4 E\left(\Delta \Delta G^{\ddagger}-E\right)} \sinh ^{2}\left(\frac{\sqrt{2 m\left(\Delta \Delta G^{\ddagger}-E\right)} Q_{C}}{\hbar}\right)\right]^{-1}
$$

and leads to $\kappa \propto 1 / \Delta \Delta G^{\ddagger}$ at very low energies, consistent with Eqn. (22).

Another significant connection is that adiabatic electron-transfer theory allows for photoinduced electron-transfer processes to be described as their occurrence stems directly from the adiabatic nature of the thermally activated electron-transfer processes. This led to the development by Hush of the theory of intervalence spectroscopy [96]. The band peak, intensity, and width information from weak intervalence transitions associated with nonadiabatic thermal processes can be interpreted to determine key properties such as the electronic coupling and reorganization energy that can then be used to interpret the observed ground-state rate constants [6, 96, 97]. Key early results [96] for the non-adiabatic regime in which the intervalence transitions are weak include

$$
\begin{aligned}
& \lambda \approx h \bar{\nu} \\
& J \approx \frac{-M}{e R} \lambda \\
& \Delta \mu \approx e R
\end{aligned}
$$

where $\bar{v}$ is the average absorption frequency (typically close to that of the absorption maximum) of the intervalence band, $M$ its transition moment, $\Delta \mu$ the dipole moment change induced by the transition, and $R$ the effective distance through which the electron is transferred,

$$
e^{2} R^{2}=(\Delta \mu)^{2}+4 M^{2}
$$

However, in general [98] for arbitrary values of the parameters it is true that

$$
J=\frac{-M}{e R} h \bar{v}
$$

whilst

$$
\lambda=h \bar{v}
$$

for all symmetric reactions $\left(\Delta G_{0}=0\right)$ in double wells $(2|J| / \lambda<1$ ) (alternatively, $h \bar{v}=2|J|$ in all symmetric delocalized situations whilst $M=e R$ ) [30]. Other general results are also known [30, 98-100]. Of particular significance is the modern re-invention of Stark spectroscopy by Steven Boxer [45, 101-104], developing experimental methods for directly measuring $\Delta \mu$ in a wide range of chemical and biochemical electron-transfer systems. These 
advances form the basis of the Generalized Mulliken-Hush method introduced by Cave and Newton for surface diabatization [105]. Nevertheless, in the weak-coupling limit, inserting Eqn. (26) into Eqn. (12) gives a simple expression for the cusp diameter in terms of easily determinable geometric and spectroscopic properties:

$$
Q_{C}=2\left|\frac{M}{\Delta \mu}\right| Q_{m} .
$$

Levich and Dogonadze's transmission coefficient can then be rewritten in terms of direct experimental observables as

$$
\kappa=4 \pi^{3 / 2}\left(\frac{h \bar{\nu}}{k_{\beta} T}\right)^{1 / 2} \frac{M^{2} Q_{m}^{2}}{\Delta \mu^{2}}
$$

Also, the exponential factor in the Levich-Dogonadze equation can be simply understood in terms of the amount of charge transferred from donor to acceptor adiabatically at the transition state [19]. In general [12, 19], the activation energy (Eqn. (3)) can be expressed without approximation in the form

$$
\Delta G^{\ddagger}=\alpha^{2} \lambda
$$

where $\alpha$ is the Tafel coefficient, deduced by Hush $[19,20]$ to be

$$
\alpha=\frac{1}{2}\left(1+\frac{\Delta G_{0}}{\lambda}\right)
$$

In the weak coupling limit for systems with $\left|\Delta G_{0}\right| \ll \lambda$ appropriate for electrochemical processes with small overpotentials, the Tafel coefficient becomes $\alpha \approx q^{\ddagger}=b^{2}\left(Q_{X}\right)$. Hence in this regime $\alpha$ is usually interpreted as the charge $q^{\ddagger}$ transferred at the geometry of the transition state [19, 20, 106]. Naively, in electrochemical situations $\alpha=1 / 2$ had been expected, and understanding its deviation with increasing overpotential through Eqn. (33) was a significant advance. These features were first highlighted by Hush in 1956 at the Moscow Conference of the Russian Electrochemical Society chaired by A. N. Frumkin [20]. Expressed in the wider context of chemical reaction dynamics (as initially envisaged by London [23]), Eqn. (33) is an expression of the Hammond-Leffler postulate [107, 108], with the cusp diameter $Q_{C}$ quantifying key variables in the revealing Reaction Force model of chemical reactivity [109-112].

More complex is the physical interpretation of the Tafel coefficient $\alpha$ for nonelectrochemical situations like the "inverted" region in which $\left|\Delta G_{0} / \lambda\right|>1$, making $\alpha$ either $<0$ or else $>1$ (Eqn. (33)), both unphysical results for the charge density at the crossover point. The Stark effect is induced by applying an external electric field $\mathbf{F}$ to an electron 
transfer process is which the total system dipole change is given in full form as a vector $\Delta \boldsymbol{\mu}$. The change in free energy of the reaction in the presence of the electric field is simply

$$
\Delta G_{0}(\mathbf{F})=\Delta G_{0}-\Delta \mu . \mathbf{F}
$$

so that the energy at the crossing point of the diabatic surfaces from Eqn. (32) is

$$
\Delta G^{\ddagger}(\mathbf{F})=\alpha^{2} \lambda-\alpha \Delta \boldsymbol{\mu} . \mathbf{F}
$$

For small free-energy differences $\left(\left|\Delta G_{0} / \lambda\right| \ll 1\right), \alpha \Delta \mu$ can be approximated by $q^{\ddagger} \Delta \boldsymbol{\mu}$ and is just the change in dipole moment between the transition-state structure and the reactants, with Eqn. (35) then indicating a linear Stark-effect response. In general, however, the Stark effect generates non-linear responses of system properties to the electric field strength. As a result, large polarizabilities and hyperpolarizabilities, as well as widely varying responses to electric fields, can be displayed by electron-transfer systems [98, 113-116]. Hence the physical interpretation of the Tafel coefficient is as the charge transferred at the crossover geometry in a simplistic model assuming that observed properties respond only linearly to an applied electric field $[12,19]$. When considering the interaction of systems at the crossover geometry with their environment, the actual charge $q^{\ddagger}=b^{2}\left(Q_{X}\right)$ should always be used.

Combining all of these results together, we see that electron-transfer reactions slow down exponentially as the amount of charge transferred at the transition state increases (Eqn. (32)), and they slow down with the inverse square of the rate at which the charge actually transfers as the crossover region is passed (Eqn. (21)).

\section{CONCLUSIONS}

The seminal equation of Levich and Dogonadze has had a profound impact on electron transfer theory and hence a large amount of modern biochemistry, chemistry, and nanotechnology. Its origins come from the Landau-Zener perturbation theories $[117,118]$ as summarized in Fermi's Golden Rule [8] that assume species are isolated and disconnected. In reality, the connection is important and we show how their simple and informative equations relate to general models of adiabatic and non-adiabatic chemical and spectroscopic processes [24, 119]. From the perspective of general adiabatic electron-transfer theory, electron-transfer rates are slowed below traditional expectations based on transition-state theory as the diagonal correction to the Born-Oppenheimer approximation leads to BornHuang adiabatic potential-energy surfaces that contain an unexpected high but narrow spike at or near the transition state. Tunnelling through this spike controls the rate of electrontransfer reactions. Electron-transfer theory is therefore unified with general expectations associated with a pitchfork bifurcation cusp catastrophe and with general properties of BornOppenheimer breakdown.

\section{REFERENCES}


1. Levich, V. G., Dogonadze, R. R. Theory of rediationless electron transitions between ions in solution // Dokl. Akad. Nauk. SSSR Ser. Fiz. Khim. 1959. V. 124. P. 123 English translation: Proc. Akad. Naukl. SSSR, 1959, vol. 24 p. 9-13.

2. Levich, V. G., Dogonadze, R. R. Adiabatic theory for electron-transfer processes in solution // Dokl. Akad. Nauk. SSSR Ser. Fiz. Khim. 1960. V. 133. P. 158 English translation: Proc. Akad. Naukl. SSSR, 1960, vol. 133 p. 156-180.

3. Levich, V. G., Dogonadze, R. R. Adiabatic theory of electron-transfer processes in solution // Collect. Czech. Chem. Commun. 1961. V. 26. P. 193.

4. Levich, V. G. Present state of the theory of oxidation=reduction in solution (bulk and electrode reactions) // in Advances in Electrochemistry and Electrochemical Engineering Vol. 4 // ed. by P. Delahaye. New York: Interscience Publishers, 1966, p. 249.

5. Marcus, R. A., Sutin, N. Electron transfers in chemistry and biology // Biochim. Biophys. Acta 1985. V. 811. P. 265.

6. Kuznetsov, A., Ulstrup, J., Electron Transfer in Chemistry and Biology: An introduction to the theory //. Chichester: Wiley, 1998, p.

7. Kubo, R., Toyozawa, Y. Application of the Method of Generating Function to Radiative and Non-Radiative Transitions of a Trapped Electron in a Crystal // Prog. Theor. Phys. 1955. V. 13. P. 160.

8. Dirac, P. A. M. The Quantum Theory of the Emission and Absorption of Radiation // Proceedings of the Royal Society of London. Series A 1927. V. 114. P. 243.

9. Libby, W. F. Theory of Electron Exchange Reactions in Aqueous Solution // J. Phys. Chem. 1952. V. 56. P. 863.

10. Marcus, R. J., Zwolinski, B. J., Eyring, H. The Electron Tunnelling Hypothesis for Electron Ex-change Reactions // J. Phys. Chem. 1954. V. 58. P. 432.

11. Weiss, J. On the Theory of Electron-Transfer Processes in Aqueous Solutions // Proc. R. Soc. London, Ser. A 1954. V. 222. P. 128.

12. Marcus, R. A. On the Theory of Oxidation-Reduction Reactions Involving Electron Transfer. 1 // J. Chem. Phys. 1956. V. 24. P. 966.

13. Holstein, T. Studies of polaron motion: Part II. The "small" polaron // Annals of Physics 1959. V. 8. P. 343.

14. Holstein, T. Studies of polaron motion: Part I. The molecular-crystal model // Annals of Physics 1959. V. 8. P. 325.

15. Lax, M. The Franck - Condon Principle and Its Application to Crystals // J. Chem. Phys. 1952. V. 20. P. 1752.

16. Mielke, S. L., Tawa, G. J., Truhlar, D. G., Schwenke, D. W. Quantum photochemistry. Accurate quantum scattering calculations for an electronically nonadiabatic reaction // Chem. Phys. Lett. 1995. V. 234. P. 57.

17. Polanyi, J. C., Zewail, A. H. Direct Observation of the Transition State // Accounts of Chemical Research 1995. V. 28. P. 119.

18. Reimers, J. R. The importance of motions that accompany those occurring along the reaction coordinate // Aust. J. Chem. 2015. V. 68. P. 1202.

19. Hush, N. S. Adiabatic rate processes at electrodes // J. Chem. Phys. 1958. V. 28. P. 962.

20. Hush, N. S. // in Soviet electrochemistry: Proceedings of the Fourth Conference on Electrochemistry 1956 // ed. by A. N. Frumkin. New York: Consultants Bureau, 1961, p. 99.

21. Delahay, P., Advances in electrochemistry and electrochemical engineering : volume 4 Electrochemistry //. New York,N.Y.: Interscience, 1966, p. 
22. Reimers, J. R., McKemmish, L., McKenzie, R. H., Hush, N. S. Non-adiabatic effects in thermochemistry, spectroscopy and kinetics: the general importance of all three Born-Oppenheimer breakdown corrections // Phys. Chem. Chem. Phys. 2015. V. 17. P. 24640.

23. London, F. Zur Quantentheorie der homöopolaren Valenzzahlen // Z. Phys. 1928. V. 46. P. 455.

24. London, F. On the theory of non-adiabatic chemical reactions // Z. Phys. 1932. V. 74. P. 143.

25. Eyring, H., Polanyi, M. Concerning simple gas reactions // Z. Phys. Chem. Abt. B 1931. V. 12. P. 279.

26. Evans, M. G., Polanyi, M. Inertia and driving force of chemical reactions // Trans. Faraday Soc. 1938. V. 34. P. 11.

27. Horiuti, J., Polanyi, M. Outlines of a theory of proton transfer // J. Molec. Catalysis A 2003. V. 199. P. 185 Translation of Acta Physicochimica U.R.S.S. 1935, 2, 505-532.

28. Wall, F. T., Glockler, G. The Double Minimum Problem Applied to the Ammonia Molecules // J. Chem. Phys. 1937. V. 5. P. 314.

29. Hush, N. S. Quantum-mechanical discussion of the gas phase formation of quinonedimethide monomers // J. Polymer Sci. 1953. V. 11. P. 289.

30. Reimers, J. R., McKemmish, L., McKenzie, R. H., Hush, N. S. A unified diabatic description for electron transfer reactions, isomerization reactions, proton transfer reactions, and aromaticity // Phys. Chem. Chem. Phys. 2015. V. 17. P. 24598.

31. Schmickler, W. A theory of adiabatic electron-transfer reactions // Journal of Electroanalytical Chemistry and Interfacial Electrochemistry 1986. V. 204. P. 31.

32. Hush, N. S. Electron transfer in retrospect and prospect 1: adiabatic electrode processes // J. Electroanal. Chem. 1999. V. 460. P. 5.

33. Reimers, J. R., McKemmish, L., McKenzie, R. H., Hush, N. S. Bond angle variations in $\mathrm{XH} 3[\mathrm{X}=\mathrm{N}, \mathrm{P}, \mathrm{As}, \mathrm{Sb}, \mathrm{Bi}]$ : the critical role of Rydberg orbitals exposed using a diabatic state model // Phys. Chem. Chem. Phys. 2015. V. 17. P. 24618.

34. McKemmish, L. K., McKenzie, R. H., Hush, N. S., Reimers, J. R. Quantum entanglement between electronic and vibrational degrees of freedom in molecules // J. Chem. Phys. 2011. V. 135. P. 244110.

35. McKemmish, L., McKenzie, R. H., Hush, N. S., Reimers, J. R. Electron-vibration entanglement in the Born-Oppenheimer description of chemical reactions and spectroscopy // Phys. Chem. Chem. Phys. 2015. V. 17. P. 24666.

36. Born, M., Oppenheimer, R. Zur Quantentheorie der Molekeln // Ann. Phys. 1927. V. 84. P. 457.

37. Born, M., Huang, K., Dynamical Theory of Crystal Lattices //. Oxford: Clarendon, 1954, p.

38. Ballhausen, C. J., Hansen, A. E. Electronic Spectra // Annu. Rev. Phys. Chem. 1972. V. 23. P. 15.

39. Hush, N. S. Electron delocalization, structure and dynamics in mixed-valence systems // NATO Adv. Study Inst. Ser., Ser. C 1980. V. 58. P. 151.

40. Marcus, R. A. Chemical and electrochemical electron-transfer theory // Annu. Rev. Phys. Chem. 1964. V. 15. P. 155.

41. Shi, Z., Boyd, R. J. Charge development at the transition state: a second-order Moeller-Plesset perturbation study of gas-phase SN2 reactions // J. Am. Chem. Soc. 1991. V. 113. P. 1072.

42. Williams, A. Effective Charge and Transition-state Structure in Solution // in Adv. Phys. Org. Chem. // ed. by D. Bethell Academic Press, 1992, p. 1. 
43. Aragonès, A. C., Haworth, N. L., Darwish, N., Ciampi, S., Bloomfield, N. J., Wallace, G. G., Diez-Perez, I., Coote, M. L. Electrostatic catalysis of a Diels-Alder reaction // Nature 2016. V. 531. P. 88.

44. Shaik, S., Mandal, D., Ramanan, R. Oriented electric fields as future smart reagents in chemistry // Nat Chem 2016. V. 8. P. 1091.

45. Wu, Y., Boxer, S. G. A Critical Test of the Electrostatic Contribution to Catalysis with Noncanonical Amino Acids in Ketosteroid Isomerase // J. Am. Chem. Soc. 2016. V. 138. P. 11890.

46. Andrews, D. L., Biological Energy 5: Electron transfer theory, 2016 Institute of Physics, biologicalphysics.iop.org/cws/article/lectures/53592.

47. Levich, V. G., Dogonadze, R. R. Theory of rediationless electron transitions between ions in solution // Proc. Akad. Naukl. SSSR 1959. V. 29. P. 9 Dokl. Akad. Nauk. SSSR Ser. Fiz. Khim. (1959) vol. 124 pages 123-126.

48. Hush, N. S. Inequivalent XPS [x-ray photoelectron spectroscopy] binding energies in symmetrical delocalized mixed-valence complexes // Chem. Phys. 1975. V. 10. P. 361.

49. Öpik, U., Pryce, M. H. L. Studies of the Jahn-Teller Effect. I. A Survey of the Static Problem // Proc. R. Soc. London, A 1957. V. 238. P. 425.

50. Saunders, P. T., An introduction to catastrophe theory //. Cambridge: Cambridge University Press, 1980, p.

51. $\mathrm{Xu}, \mathrm{F}$. Application of catastrophe theory to the $\Delta \mathrm{G}^{-}$to $-\Delta \mathrm{G}$ relationship in electron transfer reactions // Z. Phys. Chem. 1990. V. 166. P. 79.

52. Krokidis, X., Silvi, B., Dezarnaud-Dandine, C., Sevin, A. Topological study, using a coupled ELF and catastrophe theory technique, of electron transfer in the $\mathrm{Li}+\mathrm{Cl} 2$ system // New J. Chem. 1998. V. 22. P. 1341.

53. Wales, D. J. A Microscopic Basis for the Global Appearance of Energy Landscapes // Science 2001. V. 293. P. 2067.

54. Tishchenko, O., Truhlar, D. G., Ceulemans, A., Nguyen, M. T. A Unified Perspective on the Hydrogen Atom Transfer and Proton-Coupled Electron Transfer Mechanisms in Terms of Topographic Features of the Ground and Excited Potential Energy Surfaces As Exemplified by the Reaction between Phenol and Radicals // J. Am. Chem. Soc. 2008. V. 130. P. 7000.

55. Bersuker, I. B., Gorinchoi, N. N., Polinger, V. Z. On the origin of dynamic instability of molecular systems // Theor. Chim. Acta 1984. V. 66. P. 161.

56. Bersuker, I. B. Pseudo-Jahn-Teller Effect - A Two-State Paradigm in Formation, Deformation, and Transformation of Molecular Systems and Solids // Chem. Rev. 2013. V. 113. P. 1351.

57. Thompson, T. C., Truhlar, D. G., Mead, C. A. On the form of the adiabatic and diabatic representation and the validity of the adiabatic approximation for $\mathrm{X}$ [sub 3] Jahn--Teller systems // J. Chem. Phys. 1985. V. 82. P. 2392.

58. Kendrick, B. K., Mead, C. A., Truhlar, D. G. Properties of nonadiabatic couplings and the generalized Born-Oppenheimer approximation // Chem. Phys. 2002. V. 277. P. 31.

59. Zhu, C., Jasper, A. W., Truhlar, D. G. Non-Born--Oppenheimer trajectories with selfconsistent decay of mixing // J. Chem. Phys. 2004. V. 120. P. 5543.

60. Jasper, A. W., Truhlar, D. G. Non-Born-Oppenheimer molecular dynamics for conical intersections, avoided crossings, and weak interactions // Adv. Ser. Phys. Chem. 2011. V. 17. P. 375.

61. Kolos, W., Wolniewicz, L. Improved Theoretical Ground-State Energy of the Hydrogen Molecule // J. Chem. Phys. 1968. V. 49. P. 404.

62. Garrett, B. C., Truhlar, D. G. Nuclear-motion corrections to Born--Oppenheimer barrier heights for chemical reactions // J. Chem. Phys. 1985. V. 82. P. 4543. 
63. Mielke, S. L., Schwenke, D. W., Peterson, K. A. Benchmark calculations of the complete configuration-interaction limit of Born--Oppenheimer diagonal corrections to the saddle points of isotopomers of the $\mathrm{H}+\mathrm{H}$ [sub 2] reaction // J. Chem. Phys. 2005. V. 122. P. 224313.

64. Azumi, T., Matsuzaki, K. WHAT DOES TERM VIBRONIC-COUPLING MEAN // Photochem. Photobiol. 1977. V. 25. P. 315.

65. Handy, N. C., Yamaguchi, Y., Schaefer Iii, H. F. The diagonal correction to the Born-Oppenheimer approximation: Its effect on the singlet--triplet splitting of $\mathrm{CH}$ [sub 2] and other molecular effects // J. Chem. Phys. 1986. V. 84. P. 4481.

66. Jensen, J. O., Yarkony, D. R. On the evaluation of non-Born--Oppenheimer interactions for Born--Oppenheimer wave functions. V. A body fixed frame approach. Applications to isotope effects on equilibrium geometries and the adiabatic correction for the X [sup 1] Sigma [sup + ] state of LiH // J. Chem. Phys. 1988. V. 89. P. 975.

67. Valeev, E. F., Sherrill, C. D. The diagonal Born--Oppenheimer correction beyond the Hartree--Fock approximation // J. Chem. Phys. 2003. V. 118. P. 3921.

68. Gauss, J., Tajti, A., Kallay, M., Stanton, J. F., Szalay, P. G. Analytic calculation of the diagonal Born-Oppenheimer correction within configuration-interaction and coupledcluster theory // J. Chem. Phys. 2006. V. 125. P. 144111.

69. Werner, H.-J., Kallay, M., Gauss, J. The barrier height of the F + H2 reaction revisited: Coupled-cluster and multireference configuration-interaction benchmark calculations // J. Chem. Phys. 2008. V. 128. P. 034305/1.

70. Mohallem, J. R., Coura, T. d. O., Diniz, L. G., de, C. G., Assafrao, D., Heine, T. Adiabatic Corrections to Density Functional Theory Energies and Wave Functions // J. Phys. Chem. A 2008. V. 112. P. 8896.

71. Mohallem, J. R. Semiempirical evaluation of post-Hartree-Fock diagonal-BornOppenheimer corrections for organic molecules // J. Chem. Phys. 2008. V. 128. P. 144113/1.

72. Tajti, A., Szalay, P. G., Gauss, J. Perturbative treatment of the electron-correlation contribution to the diagonal Born-Oppenheimer correction // J. Chem. Phys. 2007. V. 127. P. 014102/1.

73. Crawford, T. D., Sherrill, C. D., Valeev, E. F., Fermann, J. T., King, R. A., Leininger, M. L., Brown, S. T., Janssen, C. L., Seidl, E. T., Kenny, J. P., Allen, W. D. PSI3: an open-source ab initio electronic structure package // J. Comput. Chem. 2007. V. 28. P. 1610.

74. Harding, M. E., Klopper, W. Benchmarking the Lithium-Thiophene Complex // ChemPhysChem 2013. V. 14. P. 708.

75. Przybytek, M., Jeziorski, B. Long-range asymptotic expansion of the diagonal BornOppenheimer correction // Chem. Phys. 2012. V. 401. P. 170.

76. Pavanello, M., Adamowicz, L., Alijah, A., Zobov, N. F., Mizus, I. I., Polyansky, O. L., Tennyson, J., Szidarovszky, T., Csaszar, A. G. Calibration-quality adiabatic potential energy surfaces for H3+ and its isotopologues // J. Chem. Phys. 2012. V. 136. P. 184303/1.

77. DeYonker, N. J., Allen, W. D. Taming the low-lying electronic states of FeH // J. Chem. Phys. 2012. V. 137. P. 234303/1.

78. Bozkaya, U., Turney, J. M., Yamaguchi, Y., Schaefer, H. F., III The lowest-lying electronic singlet and triplet potential energy surfaces for the HNO-NOH system: Energetics, unimolecular rate constants, tunneling and kinetic isotope effects for the isomerization and dissociation reactions // J. Chem. Phys. 2012. V. 136. P. 164303/1. 
79. Yachmenev, A., Yurchenko, S. N., Ribeyre, T., Thiel, W. High-level ab initio potential energy surfaces and vibrational energies of H2CS // J. Chem. Phys. 2011. V. 135. P. 074302/1.

80. Rao, T. R., Mahapatra, S. Nuclear motion on the orbitally degenerate electronic ground state of fully deuterated triatomic hydrogen // J. Chem. Phys. 2011. V. 134. P. 204307/1.

81. Meier, P., Neff, M., Rauhut, G. Accurate Vibrational Frequencies of Borane and Its Isotopologues // J. Chem. Theory Comput. 2011. V. 7. P. 148.

82. Lievin, J., Demaison, J., Herman, M., Fayt, A., Puzzarini, C. Comparison of the experimental, semi-experimental and ab initio equilibrium structures of acetylene: Influence of relativistic effects and of the diagonal Born-Oppenheimer corrections // J. Chem. Phys. 2011. V. 134. P. 064119/1.

83. Holka, F., Szalay, P. G., Fremont, J., Rey, M., Peterson, K. A., Tyuterev, V. G. Accurate ab initio determination of the adiabatic potential energy function and the Born-Oppenheimer breakdown corrections for the electronic ground state of $\mathrm{LiH}$ isotopologues // J. Chem. Phys. 2011. V. 134. P. 094306/1.

84. Klopper, W., Bachorz, R. A., Tew, D. P., Hattig, C. Sub-meV accuracy in firstprinciples computations of the ionization potentials and electron affinities of the atoms H to Ne // Phys. Rev. A: At., Mol., Opt. Phys. 2010. V. 81. P. 022503/1.

85. Karton, A., Martin, J. M. L. Performance of W4 theory for spectroscopic constants and electrical properties of small molecules // J. Chem. Phys.. 2010. V. 133. P. 144102.

86. Bozkaya, U., Turney, J. M., Yamaguchi, Y., Schaefer, H. F., III The barrier height, unimolecular rate constant, and lifetime for the dissociation of HN2 // J. Chem. Phys. 2010. V. 132. P. 064308/1.

87. Mielke, S. L., Schwenke, D. W., Schatz, G. C., Garrett, B. C., Peterson, K. A. Functional Representation for the Born-Oppenheimer Diagonal Correction and BornHuang Adiabatic Potential Energy Surfaces for Isotopomers of H3 // J. Phys. Chem. A 2009. V. 113. P. 4479.

88. Hobson, S. L., Valeev, E. F., Csaszar, A. G., Stanton, J. F. Is the adiabatic approximation sufficient to account for the post-Born-Oppenheimer effects on molecular electric dipole moments? // Mol. Phys. 2009. V. 107. P. 1153.

89. Hirata, S., Miller, E. B., Ohnishi, Y.-y., Yagi, K. On the Validity of the BornOppenheimer Separation and the Accuracy of Diagonal Corrections in Anharmonic Molecular Vibrations // J. Phys. Chem. A 2009. V. 113. P. 12461.

90. Schwenke, D. W. Beyond the Potential Energy Surface: Ab initio Corrections to the Born-Oppenheimer Approximation for H2O // J. Phys. Chem. A 2001. V. 105. P. 2352.

91. Newton, M. D., Sutin, N. Electron transfer reactions in condensed phases // Annu. Rev. Phys. Chem. 1984. V. 35. P. 437.

92. Jasper, A. W., Kendrick, B. K., Mead, C. A., Truhlar, D. G. Non-Born-Oppenheimer chemistry: potential surfaces, couplings, and dynamics // Adv. Ser. Phys. Chem. 2004. V. 14. P. 329.

93. Lee, S.-H., Larsen, A. G., Ohkubo, K., Cai, Z.-L., Reimers, J. R., Fukuzumi, S., Crossley, M. J. Long-lived long-distance photochemically induced spin-polarized charge separation in $\beta, \beta$ '-pyrrolic fused ferrocene-porphyrin-fullerene systems // Chem. Sci. 2012. V. 3. P. 257.

94. Yin, S., Li, L., Yang, Y., Reimers, J. R. Challenges for the Accurate Simulation of Anisotropic Charge Mobilities through Organic Molecular Crystals: The $\beta$ Phase of 
mer-Tris(8-hydroxyquinolinato)aluminum(III) (Alq3) Crystal // J. Phys. Chem. C 2012. V. 116. P. 14826.

95. Norton, J. E., Brédas, J. L. Polarization energies in oligoacene semiconductor crystals // J. Am. Chem. Soc. 2008. V. 130. P. 12377.

96. Hush, N. S. Intervalence-transfer absorption. II. Theoretical considerations and spectroscopic data // Prog. Inorg. Chem. 1967. V. 8. P. 391.

97. Kjaer, A. M., Ulstrup, J. Solvent bandwidth dependence and band asymmetry features of charge-transfer transitions in N-pyridinium phenolates // J. Am. Chem. Soc. 1987. V. 109. P. 1934.

98. Reimers, J. R., Hush, N. S. Electronic properties of transition-metal complexes determined from electro-absorption spectroscopy II. mono-nuclear complexes of ruthenium(II) // J. Phys. Chem. 1991. V. 95. P. 9773.

99. Reimers, J. R., Hush, N. S. The effects of couplings to symmetric and antisymmetric modes and minor asymmetries on the spectral properties of mixed-valence and related charge-transfer systems // Chem. Phys. 1996. V. 208. P. 177.

100. Reimers, J. R., Hush, N. S. Hamiltonian operators including both symmetric and antisymmetric vibrational modes for vibronic-coupling and intervalence chargetransfer applications // Chem. Phys. 2004. V. 299. P. 79.

101. Lockhart, D. J., Boxer, S. G. Electric Field Modulation of the Fluorescence Spectrum from Rhodobacter Sphaeroides Reaction Centers // Chem. Phys. Lett. 1988. V. 144. P. 243.

102. Boxer, S. G., Goldstein, R. A., Lockhart, D. J., Middendorf, T. R., Takiff, L. Excited states, electron-transfer reactions, and intermediates in bacterial photosynthetic reaction centers // J. Phys. Chem. 1989. V. 93. P. 8280.

103. Fried, S. D., Bagchi, S., Boxer, S. G. Extreme electric fields power catalysis in the active site of ketosteroid isomerase // Science 2014. V. 346. P. 1510.

104. Oh, D. H., Sano, M., Boxer, S. G. Electroabsorption (Stark effect) spectroscopy of mono- and biruthenium charge-transfer complexes: measurements of changes in dipole moments and other electrooptic properties // J. Am. Chem. Soc. 1991. V. 113. P. 6880.

105. Cave, R. J., Newton, M. D. Generalization of the Mulliken-Hush treatment for the calculation of electron transfer matrix elements // Chem. Phys. Lett. 1996. V. 249. P. 15.

106. Hush, N. S. Discussion of "Electrode reactons of organic compounds: General Introduction" by R.A. Marcus // Discussions of the Faraday Society 1968. V. 45. P. 52.

107. Hammond, G. S. A correlation of reaction rates // J. Am. Chem. Soc. 1955. V. 77. P. 334.

108. Leffler, J. E. Parameters for the description of transition states // Science 1953. V. 117. P. 340.

109. Toro-Labbe, A. Characterization of Chemical Reactions from the Profiles of Energy, Chemical Potential, and Hardness // J. Phys. Chem. A 1999. V. 103. P. 4398.

110. Toro-Labbe, A., Gutierrez-Oliva, S., Murray, J. S., Politzer, P. A new perspective on chemical and physical processes: the reaction force // Mol. Phys. 2007. V. 105. P. 2619.

111. Toro-Labbe, A., Gutierrez-Oliva, S., Murray, J. S., Politzer, P. The reaction force and the transition region of a reaction // J. Mol. Model. 2009. V. 15. P. 707.

112. Politzer, P., Reimers, J. R., Murray, J. S., Toro-Labbe, A. Reaction Force and Its Link to Diabatic Analysis: A Unifying Approach to Analyzing Chemical Reactions // J. Phys. Chem. Lett. 2010. V. 1. P. 2858. 
113. Kanchanawong, O., Dahlbom, M. G., Treynor, T. P., Reimers, J. R., Hush, N. S., Boxer, S. G. Charge Delocalization in the Special Pair Radical Cation of Mutant Reaction Centers of Rhodobacter Sphaeroides from Stark Spectra and Non-Adiabatic Spectral Simulations // J. Phys. Chem. B 2006. V. 110. P. 18688.

114. Reimers, J. R., Hush, N. S. Understanding the observed Stark spectra, midpoint potential versus degree of charge localization, and intervalence transition energies of the special-pair radical cation of Rhodobacter sphaeroides and its mutant strains // J. Am. Chem. Soc. 2004. V. 126. P. 4132.

115. Reimers, J. R., Hush, N. S. Electric field perturbation of electronic (vibronic) absorption envelopes: Application to characterization of mixed-valence states // in Mixed Valence Systems: Applications in Chemistry, Physics, and Biology // ed. by K. Prassides. Dordrecht: Kluwer Acad. Publishers, 1991, p. 29.

116. Franzen, S., Lao, K., Boxer, S. G. Electric field effects on kinetics of electron transfer reactions: connection between experiment and theory // Chem. Phys. Lett. 1992. V. 197. P. 380.

117. Landau, L. D. // Z. Phys. Sowjetunion 1932. V. 2. P. 46.

118. Landau, L. D. // Z. Phys. Sowjetunion 1932. V. 1. P. 88.

119. London, F. Ueber den Mechanismus der homöopolaren Bindung. // in Probleme der Modernen Physik // ed. by A. Sommerfeld and P. Debye. Leipzig: Hirsel, 1928, p. 104. 


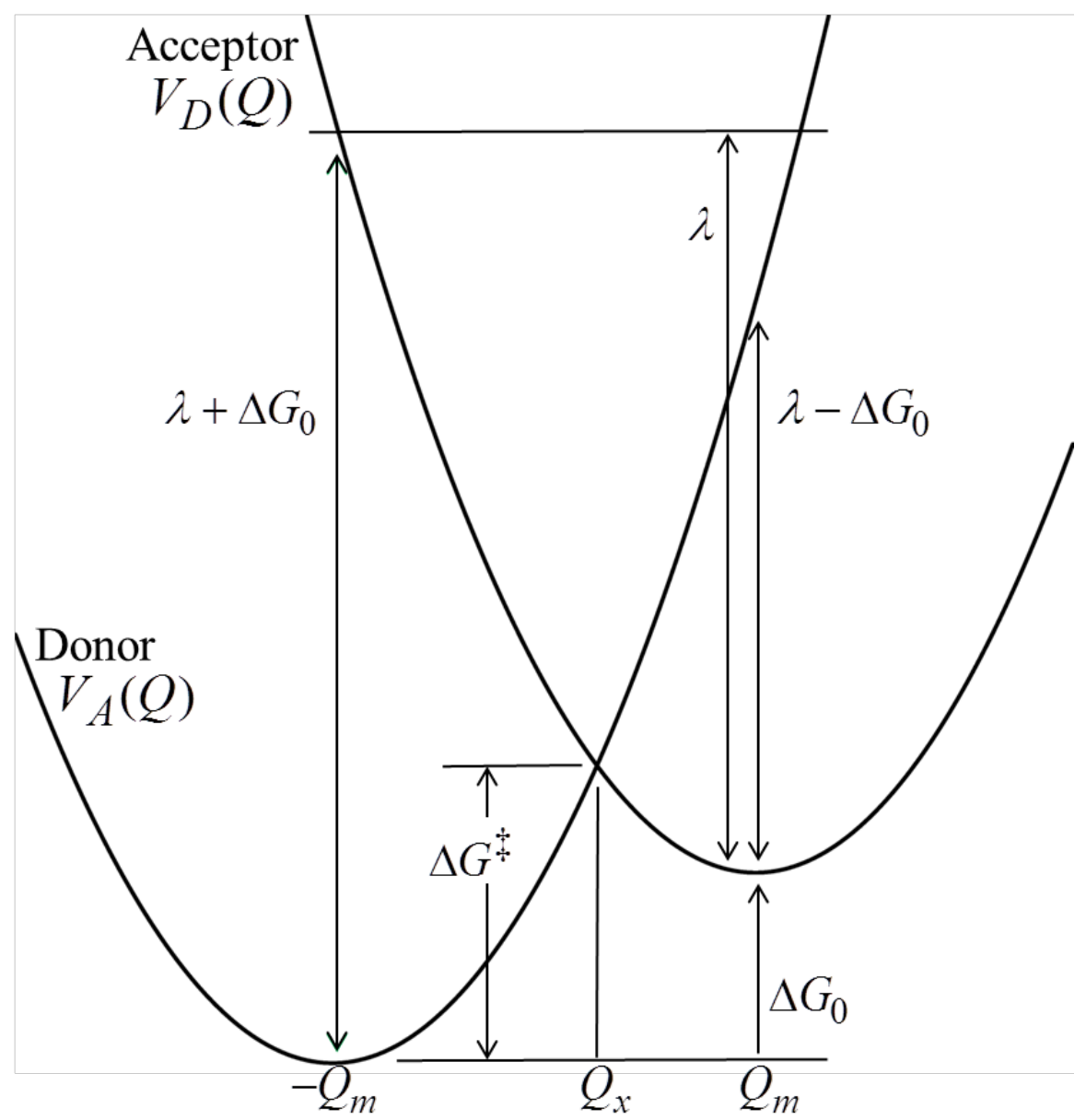

Figure 1. Properties of the diabatic description (Eqn. (7)) of electron-transfer reactions in the limit of very weak coupling. 


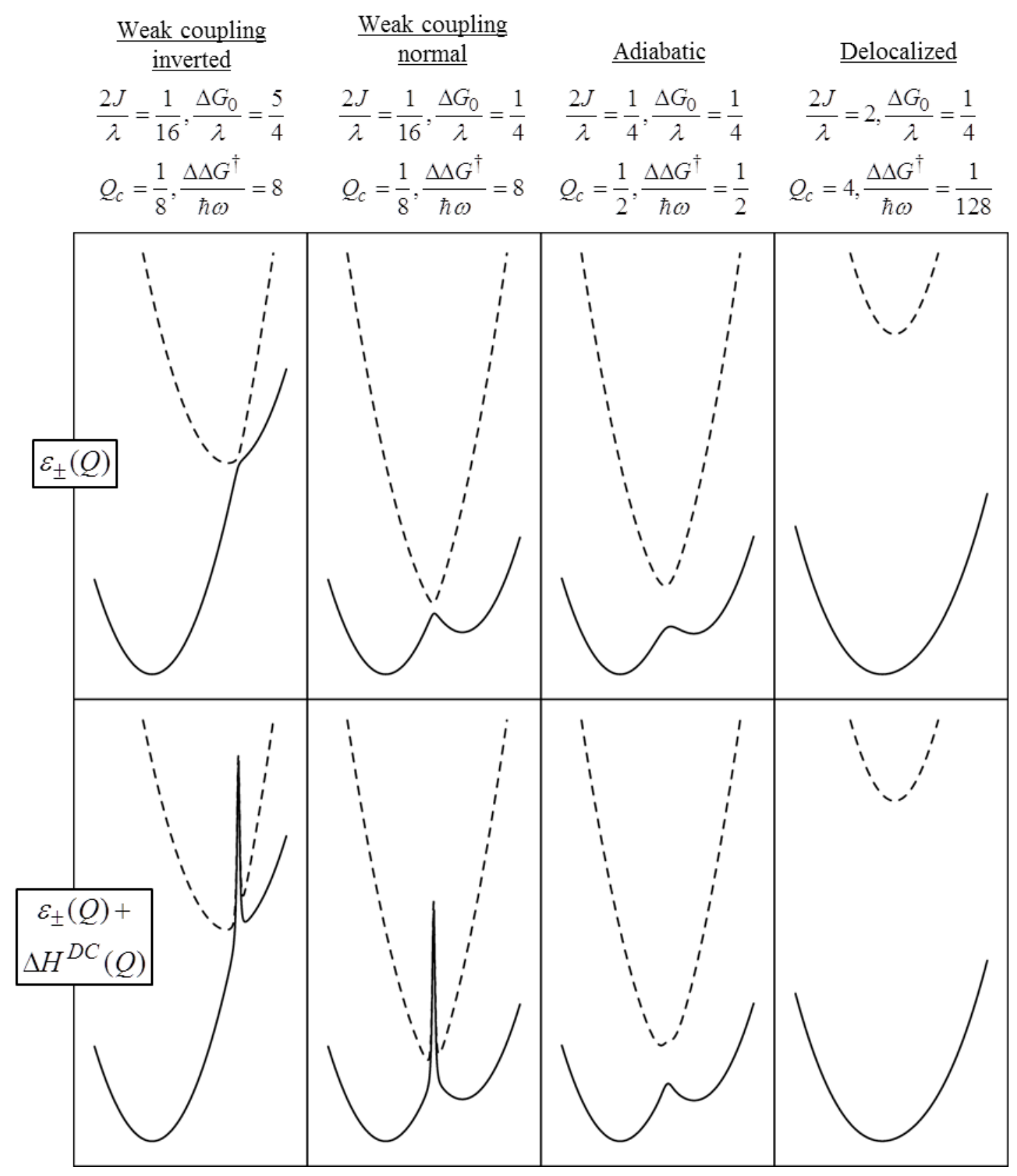

Figure 2. The top frames show the Born-Oppenheimer adiabatic potential-energy surfaces $\varepsilon_{ \pm}(Q)$ (ground state solid lines, excited state dashed lines), while the bottom frames show the corresponding Born-Huang adiabatic potential-energy surfaces $\varepsilon_{ \pm}^{\mathrm{BH}}(Q)=\varepsilon_{ \pm}(Q)+\Delta H^{D C}(Q)$, for situations in the weak-coupling limit (both the "inverted" and "normal” regions), adiabatic regime, and delocalized regimes. In all examples $Q_{m}=2$ and $\lambda=8 \hbar \omega$. 


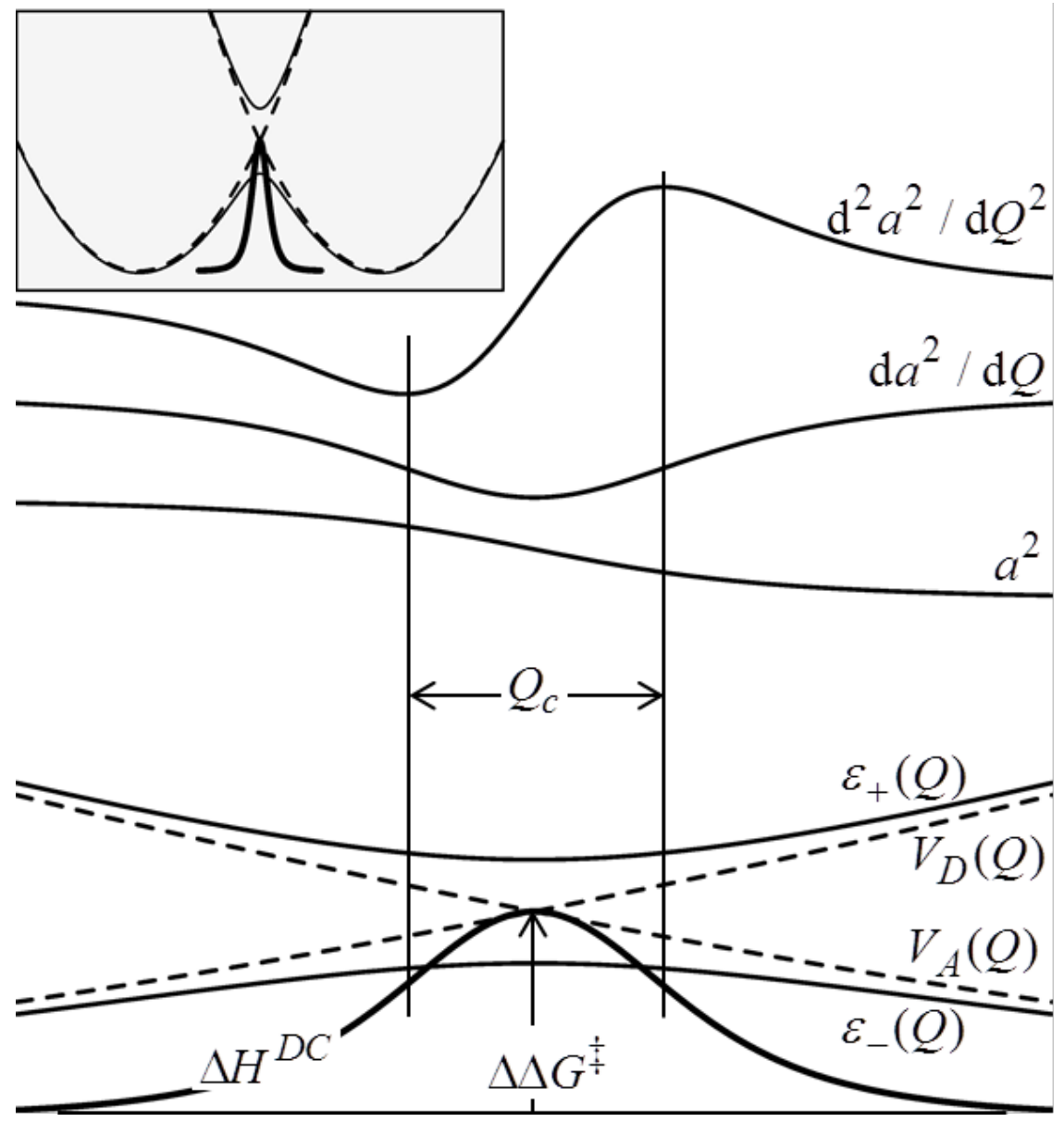

Figure 3. The lower curves (and insert) show the potential-energy surfaces for electron transfer with $2 J / \lambda=1 / 8, \Delta G_{0}=0, \lambda=8 \hbar \omega$ so that $Q_{C}=1 / 4$ and $\Delta \Delta G^{\ddagger}=2 \hbar \omega:$ dashed lines- diabatic donor $V_{D}(Q)$ and acceptor $V_{A}(Q)$ surfaces (Eqn. (7)), thin solid lines- BornOppenheimer adiabatic surfaces $\varepsilon_{ \pm}(Q)$ (Eqn.(8)), thick solid lines- the diagonal correction to the Born-Oppenheimer surfaces $\Delta H^{D C}(Q)$ (Eqn. (16)). The upper curves show properties coming from the gradual change in the nature of the ground state surface as the transitionstate region is crossed: $a^{2}$ (Eqn. (10)) gives the probability that the ground-state is donor-like (it changes from $\sim 1$ to $\sim 0$ as $Q$ increases), with its first and second derivatives also shown. 